\title{
Vaccination timeliness and associated factors among children aged 12-23 months in northwest Ethiopia: community-based study
}

\section{Bekalu Endalew}

Jabitehnan District Health Office

\section{Ayal Debie}

University of Gondar

Lake Yazachew ( $\sim$ lyazachew07@gmail.com)

University of Gondar https://orcid.org/0000-0001-8863-1701

\section{Research}

Keywords: vaccination timeliness, Children, Ethiopia

Posted Date: August 17th, 2020

DOI: https://doi.org/10.21203/rs.3.rs-56134/v1

License: (c) (i) This work is licensed under a Creative Commons Attribution 4.0 International License.

Read Full License 


\section{Abstract}

Background: vaccines are the most effective preventive and success of public health to control and eradicate serious childhood diseases. Timely childhood vaccination can help for children to develop antibody against vaccine preventable diseases. Evidences on childhood vaccination timeliness, however, there are limited in developing countries including Ethiopia. Therefore, this study was aimed at assessing vaccination timeliness and associated factors among children aged 12 to 23 months in Jabitehnan district, northwest Ethiopia.

Methods: A community based cross-sectional study was conducted in Jabitehnan district from February to March 2020. A total of 548 children aged 12-23 months were included using multi-stage and simple random sampling technique. Binary logistic regression model was fitted to identify factors associated with vaccination timeliness. Adjusted odd ratio (AOR) with $95 \% \mathrm{Cl}$ and $p$-value less than 0.05 were used to declare statistically significant variables.

Results: A total of $13.1 \%$ (95\% Cl: 10.1-15.8) of children were received childhood vaccines in the recommended time interval. Higher level of maternal education (AOR: 2.73; 95\% Cl: 1.14-6.50), history of abortion (AOR: 3.45 ; 95\% Cl: 1.54-7.74), knowledge (AOR: 1.79; 95\% Cl: 1.10-3.18) and favorable attitude (AOR: $3.38 ; 95 \% \mathrm{Cl}$ : $1.83-6.24$ ) were positively associated with vaccination timeliness. While home delivery (AOR: $0.35 ; 95 \% \mathrm{Cl}: 0.18-0.68$ ) and rural residence (AOR: $0.31 ; 95 \% \mathrm{Cl}: 0.14-0.65)$ were negatively associated.

Conclusion: The overall childhood vaccination timeliness status was low in the study area. Children were received the first dose of vaccines within the recommended time relatively higher than the other doses/vaccines. Moreover, children were received measles vaccine earlier and BCG vaccine later than the acceptable time interval. Variables such as residence, maternal level of education, having history of abortion, place of delivery, knowledge and attitudes were affecting vaccination timeliness. Therefore, the policy planners and managers should give emphasis and incorporate vaccination timeliness in the childhood vaccination plan, and better to monitor and evaluate as one potential indicator to enhance the immune status of children. Health planners and managers should also improve women's awareness to enhance their attitude towards childhood vaccination. Furthermore, it is better to promote institutional delivery service utilization to enhance childhood vaccination timeliness.

\section{Introduction}

Immunization is the process by which a person is made immune or resistant to an infectious disease, typically by the administration of a vaccine (1). The Expanded Program on Immunization (EPI) was started in early 1974 to give all basic vaccines and immunize every child around the world and launched in Ethiopia since1980 (2). Immunization is a central pillar of universal health coverage (3), and one of the most effective and successful means of public health interventions to control and eradicate serious 
diseases (4). It is a highly effective strategy that helps the parents to prevent their children from major infectious diseases, sequelae, hospitalization and even death (5).

World Health Organization (WHO) recommends that vaccines must be administrated during the first year of life within a specified schedule and time range (6). Immunization timeliness is defined as the administration of vaccines at the earliest appropriate age and recommended intervals between vaccine doses (7). Vaccinating children at an appropriate time interval is an important mechanism to protect diseases adequately since early vaccination can result in failure to generate a protective antibody against the diseases and delayed vaccination end up with risk factors for vaccine preventable diseases $(8,9)$. Increased compliance to vaccine timeliness safeguards that children are protected prior to exposure and controls morbidity through improving population immunity and potential spread of communicable diseases, particularly in the form of disease outbreaks $(10,11)$.

About $86 \%$ of the children receive vaccines and prevents 2-3 million deaths every year from childhood vaccine preventable diseases in the world(12). Despite this success of childhood interventions approximately 1.5 million children died by vaccine preventable diseases each year, particularly in developing countries (13). Low and middle income countries (LMICs) encountered a disproportionately high burden of vaccine-preventable diseases(14). Every year more than 6.3 million children in low- and middle-income countries die before they reach their fifth birthdays and the leading cause of this is ineffectiveness of vaccine administration (15). In sub-Saharan African countries, vaccine preventable diseases (VPDs) are also the major contributors to high child mortality (16).

In Ethiopia, under one mortality is 40 per 1000 live births per year and the leading cause of this death is vaccine preventable diseases(17). Moreover, about 7,951 suspected measles cases and around 3.5 million children become susceptible to measles every year in the country(18).

Although childhood vaccination coverage in Ethiopia has shown improvements (more than 90\%) except measles (83\%), childhood vaccine preventable disease outbreaks and high associated childhood mortality rate are still common nationwide. It was mainly because of the failure to achieve "herdimmunity'(18). This indicates that maximizing vaccination timeliness to attain the full benefits of vaccinations is crucial and untimely vaccination coverage may mask the absolute timely vaccination coverage (19).

Childhood vaccination timeliness is low in many African countries, including Ethiopia ranged from 6.2$68 \%$ (20-22). Both early and delayed childhood vaccination have their own disadvantages i.e. early vaccination can result in failure to generate a protective antibody against the diseases while delayed vaccination end up with risk factors for vaccine preventable diseases $(8,9)$.

The high childhood mortality rate due to vaccine preventable diseases indicates that being satisfied with vaccination coverage alone can mislead to false assumption for vaccine preventable disease protection. Studies revealed that a timely childhood vaccination is a good quality indicator for the expanded program 
on immunization to protect children from vaccine preventable diseases through enhancing sero conversion rate of vaccines.

However, there are limited studies in our country on childhood vaccination timeliness. Therefore, this

study was aimed at assessing vaccination timeliness and associated factors among children aged 12 to 23 months in Jabitehnan district, northwest Ethiopia.

\section{Methods}

\section{Study design and settings}

A community based cross-sectional study design was conducted among mothers/caregivers of children aged 12 to 23 months at Jabitehnan district, northwest Ethiopia. The study was done from February to March 2020. Jabitehnan is located $180 \mathrm{Km}$ in the southwest of Bahir Dar (Capital city of Amhara National Regional State) and $387 \mathrm{Km}$ in the northwest of Addis Ababa (Capital city of Ethiopia). The estimated population of the district in 2019/2020 based upon 2007 Central Statistical Agency (CSA) of Ethiopia was 228,246 , of whom 112,982 were males and $16,045(7.03 \%)$ were urban inhabitants. It has around 30,905 under five children and around 15,526 under two children and 6163 children aged 12 to 23 months (24). The district has thirty-nine rural and three urban kebeles. On top of that, there are eleven health centers and 41 health posts in the district. All of these health facilities provide both outreach and static childhood vaccination services.

\section{Population and sampling procedures}

The source and study population were all children aged 12 to 23 months who had started vaccination and vaccination card or registered in childhood vaccination register in Jabitehnan district and selected kebeles of the district, respectively. All mothers / care givers of children aged 12 to 23 months who had started vaccination and who had a vaccination card or a vaccination register in the selected kebele were included in this study, however, those who were severely ill during data collection and children who had vaccination cards but no registration date of vaccination or date of birth either in the card or in the infant immunization register were excluded from the study.

The sample size was determined using the single population proportion formula by assuming a $6.2 \%$ proportion of children received timely vaccination study in northeast Ethiopia (22), 3\% margin of error, $95 \%$ confidence level, $10 \%$ non-response rate and 2 design effect. The final sample size was 548.

A multi-stage simple random sampling technique was used to select study participants. First the kebeles of the district were stratified to 39 rural and 03 urban. From the total of 42 kebeles in the district ten (one urban and nine rural) were selected randomly using the list of all eligible children aged 12 to 23 months from the family folder and infant immunization registers of the selected health posts. Health extension workers update the information recorded in the family folder at least once per month in every health posts. The list of all eligible children obtained from the health posts family folder were cross-checked 
with the infant immunization registers in the health posts and health centers to confirm the missing of eligible children from the sampling frame. The complete list of all eligible children in the selected kebele containing information about the name of a child, his/her parent's full name, household's unique identification number and sub-kebele/gotte/ was prepared by using family folders and infant immunization registers of the health posts and health centers. Proportional allocation of the sample was made to determine the required sample size from each selected kebele. Finally, simple random sampling technique was employed to select the required number of children from each selected kebele using the listed children as a sampling frame. The mothers/care givers of the index children were identified using the unique ID of the household in the selected kebeles.

\section{Variables and measurement}

Vaccination timeliness was the dependent variable, while socio-demographic related factors, such as age, sex of child, income, occupation, marital status, educational status of parents, residence, religion of parents, household size and birth order; access related factors: distance from vaccination site, mode of transportation, season of birth, availability of phone, availability of electronic media and place of vaccination; obstetric related factors: ANC visit, PNC visit, maternal conference participation, TT vaccination, pregnancy status, institutional delivery, birth attendance and parity; maternal awareness related factors: knowledge about vaccination and attitude towards vaccination were the independent variables.

Timely vaccination: a child is considered to be timely vaccinated, If the child received BCG within the first fifty-six days, OPV1, penta1, PCV1 and Rota1 from 39 to 70 days, OPV 2, penta2, PCV2 and Rota 2 from 67 to 98 days, OPV3, penta3 and PCV3 from 95 to 126 days and measles vaccine 270 to 301 days of age of the child $(6,22)$. On the other hand, the child was considered as early vaccinated when the child received at least one dose of the vaccine below the minimum recommended age for each antigens, and considered as delayed vaccination when the child received at least one dose of the vaccine above the maximum recommended age $(6,22)$.

Good knowledge: Twelve knowledge assessment item questions each containing ( $0=$ no and $1=y e s)$ alternatives were used and those who scored greater than $50 \%$ of the total knowledge measuring score was considered as having good knowledge (25).

Favorable attitude: Eight Likert scale attitude assessment item questions each containing ( $1=$ strongly disagree, $2=$ disagree, $3=$ neutral, $4=$ agree and $5=$ strongly agree) alternatives were used and those who scored $75 \%$ or more the attitude measuring score were considered as having favorable attitude $(22,26)$.

\section{Data collection tools and procedures}

The data were collected using interviewer administered semi-structured questionnaire. Five data collectors (nurses) and two supervisors (Health officers) were used for data collection. The questionnaire had socio-demographic, awareness, obstetric characteristics of mothers, and access related factors parts. 
The questionnaire was prepared first in English and translated to Amharic and then back to English to maintain consistency. One day training was given for both data collectors and supervisors on the basic techniques of the data collection procedures. Pre-test was conducted at Dembecha district among 28 (5\%) mothers/caregivers of children aged 12 to 23 months who had started vaccination and had a vaccination card or a list in the infant immunization register, and necessary modification was made based on the pre-test findings. Completeness and consistency of the data were checked on the spot and daily basis by the supervisors and the principal investigator.

\section{Data processing and analysis}

The data were entered, cleaned and coded using Epi data software version 4.6 and exported to Statistical Packages for Social Sciences (SPSS) (version 22.0 Software for analysis. Descriptive statistics, such as mean, median, frequency and percentage were presented using texts, graphs and tables. Binary logistic regression model was used to identify factors associated with childhood vaccination timeliness. Those independent variables with p-value less than 0.2 during bi-variable analysis were taken into multivariable logistic regression analysis (22). Adjusted odd ratio (AOR) with $95 \%$ confidence level $(95 \% \mathrm{Cl})$ and $p$-value less than 0.05 during multivariable logistic regression were used to declare statistically significant association with childhood vaccination timeliness and the strength of association.

\section{Results}

\section{Socio-demographic characteristics of participants}

A total of 543 mothers/caregivers of children aged 12 to 23 months interviewed with response rate of $99 \%$. Nearly ninety percent $(98.7 \%)$ of the participants were mothers of children and the remaining $1.7 \%$ of the respondents were caregivers. The mean age of the mothers/caregivers was 29.5 years $( \pm 5.96 \mathrm{SD})$ which ranges from 18 - 50 years. Three hundred ninety (71.8\%) of the respondents were in the age group of 25 - 34 years. Nearly $90 \%$ of the participants were rural residents and over $95 \%$ of the respondents were Orthodox Christians. Two-third of the participants were farmers, more than $90 \%$ were married and threefifth $(60 \%)$ of the participants were unable to read and write. The mean ages of the children were 18.11months ( $\pm 3.25 \mathrm{SD})$ (Table 1).

Table 1: Scio-demographic characteristics of the participants in Jabitehnan district, Ethiopia, 2020 ( $n=543)$ 


\begin{tabular}{|c|c|c|}
\hline Characteristics & Frequency & Percent $(\%)$ \\
\hline \multicolumn{3}{|c|}{ Age of mothers/caregivers (years) } \\
\hline $15-24$ & 12 & 2.2 \\
\hline $25-34$ & 390 & 71.8 \\
\hline 35 or more & 141 & 26.0 \\
\hline \multicolumn{3}{|l|}{ Sex of child } \\
\hline Male & 268 & 49.4 \\
\hline Female & 275 & 50.6 \\
\hline \multicolumn{3}{|l|}{ Age of child (months) } \\
\hline $12-18$ & 278 & 51.2 \\
\hline $19-23$ & 265 & 48.8 \\
\hline \multicolumn{3}{|l|}{ Residence } \\
\hline Urban & 55 & 10.1 \\
\hline Rural & 488 & 89.9 \\
\hline \multicolumn{3}{|l|}{ Religion } \\
\hline Orthodox & 529 & 97.4 \\
\hline Muslim & 14 & 2.6 \\
\hline \multicolumn{3}{|l|}{ Maternal education } \\
\hline Can't read and write & 345 & 63.5 \\
\hline Can read and write & 90 & 16.6 \\
\hline Primary (1-8) & 69 & 12.7 \\
\hline Secondary (9-12) and above & 39 & 7.2 \\
\hline \multicolumn{3}{|l|}{ Occupation of mothers } \\
\hline Housewife & 141 & 26.0 \\
\hline Farmer & 353 & 65.0 \\
\hline Others & 49 & 9.0 \\
\hline \multicolumn{3}{|l|}{ Current marital status } \\
\hline Married & 504 & 92.8 \\
\hline Unmarried & 39 & 7.2 \\
\hline \multicolumn{3}{|l|}{ Family size } \\
\hline$<5$ & 218 & 40.1 \\
\hline$\geq 5$ & 325 & 59.9 \\
\hline \multicolumn{3}{|l|}{ Paternal education } \\
\hline Can't read and write & 245 & 45.1 \\
\hline Can read and write & 176 & 32.4 \\
\hline Primary (1-8) & 63 & 11.6 \\
\hline Secondary (9-12) and above & 59 & 10.9 \\
\hline \multicolumn{3}{|l|}{ Paternal occupation } \\
\hline Farmer & 458 & 84.4 \\
\hline Merchant & 45 & 8.3 \\
\hline Others & 40 & 7.3 \\
\hline \multicolumn{3}{|c|}{ House hold monthly income (ETB*) } \\
\hline$<1000$ & 129 & 23.8 \\
\hline $1001-2500$ & 295 & 54.3 \\
\hline 2501 or more & 119 & 21.9 \\
\hline
\end{tabular}

*ETB: Ethiopian Birr 
Over sixty percent (61.5\%) of mothers/caregivers were attended institutional delivery and $76.6 \%$ received PNC services for their recent child. More than one-third (35.4\%) of women had 5 or more parity and $56 \%$ of respondents did not participate in maternal conference. Almost half $(48.3 \%)$ of mothers/caregivers had good knowledge and $47.3 \%$ mothers/caregivers had favorable attitude towards childhood vaccination. Sixty-nine percent of mothers/care givers had got information about childhood vaccination from health professionals (Table 2).

Table 2: Obstetric history and access to information of the participants in Jabitehnan district, Ethiopia, 2020 $(\mathrm{n}=543)$ 


\begin{tabular}{|c|c|c|}
\hline Variables & Frequency & Percent (\%) \\
\hline \multicolumn{3}{|l|}{ Pregnancy status } \\
\hline Planned & 462 & 85.1 \\
\hline Un planned & 81 & 14.9 \\
\hline \multicolumn{3}{|l|}{ Number of ANC visit } \\
\hline 0 & 68 & 12.5 \\
\hline$\leq 2$ & 141 & 26.0 \\
\hline$\geq 3$ & 334 & 61.5 \\
\hline \multicolumn{3}{|l|}{ TT dose received } \\
\hline No & 90 & 16.6 \\
\hline One & 75 & 13.8 \\
\hline two or more & 378 & 69.6 \\
\hline \multicolumn{3}{|c|}{ Maternal conference participation } \\
\hline No & 305 & 56.2 \\
\hline$\leq 2$ & 137 & 25.2 \\
\hline$\geq 3$ & 101 & 18.6 \\
\hline \multicolumn{3}{|c|}{ PNC service utilization } \\
\hline No & 127 & 23.4 \\
\hline Yes & 416 & 76.6 \\
\hline \multicolumn{3}{|l|}{ Place of delivery } \\
\hline Healthy facilities & 334 & 61.5 \\
\hline Home & 209 & 38.5 \\
\hline \multicolumn{3}{|l|}{ Mode of delivery } \\
\hline Spontaneous & 489 & 90.0 \\
\hline Caesarian section & 22 & 4.1 \\
\hline Instrumental & 32 & 5.9 \\
\hline \multicolumn{3}{|l|}{ Parity } \\
\hline$<5$ & 351 & 64.6 \\
\hline$\geq 5$ & 192 & 35.4 \\
\hline \multicolumn{3}{|l|}{ Mode of transport } \\
\hline Foot & 509 & 93.7 \\
\hline Vehicle & 34 & 6.3 \\
\hline \multicolumn{3}{|c|}{ Time taken to vaccination site (minutes) } \\
\hline$<30$ & 482 & 88.8 \\
\hline$\geq 30$ & 61 & 11.2 \\
\hline \multicolumn{3}{|l|}{ Seasons of birth } \\
\hline Summer & 169 & 31.1 \\
\hline Autumn & 131 & 24.1 \\
\hline Winter & 99 & 18.2 \\
\hline Spring & 144 & 26.6 \\
\hline \multicolumn{3}{|c|}{ Source of information } \\
\hline Mobile & 11 & 2.0 \\
\hline Television & 55 & 10.5 \\
\hline Radio & 124 & 22.8 \\
\hline Health workers & 376 & 69.2 \\
\hline \multicolumn{3}{|c|}{ Place of vaccines received } \\
\hline Health center & 153 & 28.2 \\
\hline Health post & 132 & 24.3 \\
\hline Out reach & 258 & 47.5 \\
\hline
\end{tabular}




\begin{tabular}{lll} 
Good & 262 & 48.3 \\
\hline Poor & 281 & 51.7 \\
\hline Attitude of mothers & & \\
\hline Favorable & 257 & 47.3 \\
\hline Unfavorable & 286 & 52.7 \\
\hline
\end{tabular}

\section{Childhood vaccination timeliness}

Overall, $13.1 \%$ (95\% Cl: 10.1-15.8) of children aged 12-23 months were received their vaccinations at the recommended time interval. About $18.4 \%$ (95\% Cl: $15.1-21.6)$ and $79.4 \%(95 \% \mathrm{Cl}$ : $76.2-82.7)$ of children aged 12-23 months were received their vaccinations earlier and later than the recommended time interval, respectively. About $42.2,64.3,41.4$ and $42.9 \%$ of children received BCG, Penta1, Penta3 and measles vaccines in the recommended time interval, respectively. Moreover, $5.5,2.6$ and $16.4 \%$ of children were received Penta1, Penta3 and measles vaccines earlier than the acceptable time interval, respectively. On the contrary, $53.6,30,53.0$ and $23.6 \%$ of children took BCG, Penta1, Penta3 and measles vaccines later than the acceptable time interval, correspondingly (Table 3 ).

Table 3: Vaccination timeliness among children aged 12-23 months in Jabitehnan district, northwest Ethiopia, $2020(n=543)$.

\begin{tabular}{|c|c|c|c|c|}
\hline \multirow[t]{2}{*}{ Antigens } & \multicolumn{3}{|c|}{ Vaccinated } & \multirow[t]{2}{*}{ Unvaccinated, n (\%) } \\
\hline & Early, n (\%) & Timely, n (\%) & Late, n (\%) & \\
\hline BCG & - & $229(42.2)$ & $291(53.6)$ & $23(4.2)$ \\
\hline OPV1 & $30(5.5)$ & $349(64.3)$ & $163(30.0)$ & $1(0.2)$ \\
\hline OPV2 & $16(2.9)$ & $270(49.7)$ & $251(46.3)$ & $6(1.1)$ \\
\hline OPV3 & $14(2.6)$ & $225(41.4)$ & $288(53.0)$ & $16(2.9)$ \\
\hline Penta1 & $30(5.5)$ & $349(64.3)$ & $163(30.0)$ & $1(0.2)$ \\
\hline Penta2 & $16(2.9)$ & $270(49.7)$ & $251(46.3)$ & $6(1.1)$ \\
\hline Penta3 & $14(2.6)$ & $225(41.4)$ & $288(53.0)$ & $16(2.9)$ \\
\hline PCV1 & $30(5.5)$ & $349(64.3)$ & $163(30.0)$ & $1(0.2)$ \\
\hline PCV2 & $16(2.9)$ & $270(49.7)$ & $251(46.3)$ & $6(1.1)$ \\
\hline PCV3 & $14(2.6)$ & $225(41.4)$ & $288(53.0)$ & $16(2.9)$ \\
\hline Rota1 & $30(5.5)$ & $349(64.3)$ & $163(30.0)$ & $1(0.2)$ \\
\hline Rota2 & $16(2.9)$ & $270(49.7)$ & $251(46.3)$ & $6(1.1)$ \\
\hline Measles & $89(16.4)$ & $233(42.9)$ & $128(23.6)$ & $93(17.1)$ \\
\hline All antigens & $100(18.4)$ & $71(13.1)$ & $431(79.4)$ & - \\
\hline
\end{tabular}




\section{Factors associated with childhood vaccination timeliness}

The multivariable logistic regression analysis illustrates that variables such as maternal level of education, residence, history of abortion, place of delivery, knowledge and attitude of mothers towards vaccination were remained to be significantly associated with childhood vaccination timeliness among children aged 12 to 23 months in the study area.

Accordingly, children lived in rural areas were $69 \%$ (AOR: $0.31: 95 \% \mathrm{Cl}: 0.14-0.65)$ less likely to receive vaccines at the recommended age than urban children. Mothers/caregivers who gave birth at home were $65 \%$ (AOR: $0.35 ; 95 \% \mathrm{Cl}: 0.18-0.68$ ) less likely to be vaccinated timely compared with those who gave birth at health facilities. Children of educated mothers were 2.73 times (AOR: $2.73 ; 95 \% \mathrm{Cl}$ : 1.14-6.50) more likely to receive childhood vaccination timely than mothers who can't read and write. Children of mothers who had history of abortion were 3.45 times (AOR: $3.45 ; 95 \% \mathrm{Cl}: 1.54-7.74)$ more likely to receive vaccination timely compared with their counterparts. Children of mothers/caregivers with favorable attitude towards childhood vaccination were 3.38 times (AOR: $3.38 ; 95 \% \mathrm{Cl}$ : $1.83-6.24$ ) more likely to be vaccinated timely than children of mothers with unfavorable attitude. Children of mothers/caregivers with good knowledge about childhood vaccination were 1.79 times (AOR: 1.79; 95\% Cl: 1.10-3.18) more likely to receive vaccination within the recommended time interval than their counterparts (Table 4).

Table 4: Factors associated with vaccination timeliness among children aged 12 - 23 months in Jabitehnan district, northwest Ethiopia, 2020 ( $\mathrm{n}=543)$. 


\begin{tabular}{|c|c|c|c|c|}
\hline \multirow[t]{2}{*}{ Characteristics } & \multicolumn{2}{|c|}{ Vaccination timeliness } & \multirow[t]{2}{*}{ COR $(95 \% \mathrm{CI})$} & \multirow[t]{2}{*}{ AOR (95\%CI) } \\
\hline & Yes & No & & \\
\hline \multicolumn{5}{|l|}{ Residence } \\
\hline Rural & 54 & 434 & $0.28(0.15-0.53)$ & $0.31(0.14-0.65) * *$ \\
\hline Urban & 17 & 38 & 1.00 & 1.00 \\
\hline \multicolumn{5}{|l|}{ Maternal education } \\
\hline Can't read and write & 36 & 309 & 1.00 & 1.00 \\
\hline Can read and write & 14 & 76 & $1.58(0.81-3.10)$ & $1.85(0.90-3.78)$ \\
\hline Primary (1-8) & 8 & 61 & $1.12(0.50-2.50)$ & $0.76(0.30-1.92)$ \\
\hline Secondary and above & 13 & 26 & $4.30(2.03-9.10)$ & $2.73(1.14-6.50) *$ \\
\hline \multicolumn{5}{|l|}{ Paternal education } \\
\hline Can't read and write & 22 & 223 & 1.00 & 1.00 \\
\hline Can read and write & 24 & 152 & $1.60(0.87-2.96)$ & $1.26(0.64-2.52)$ \\
\hline Primary (1-8) & 12 & 51 & $2.38(1.11-5.13)$ & $1.51(0.56-3.83)$ \\
\hline Secondary and above & 13 & 46 & $2.86(1.34-6.10)$ & $1.01(0.28-3.64)$ \\
\hline \multicolumn{5}{|l|}{ Birth order } \\
\hline First & 21 & 99 & 1.00 & 1.00 \\
\hline Second & 12 & 78 & $0.72(0.34-1.56)$ & $0.84(0.34-2.11)$ \\
\hline Three or more & 38 & 295 & $0.60(0.34-1.10)$ & $0.76(0.33-1.74)$ \\
\hline \multicolumn{5}{|l|}{ Maternal conference } \\
\hline 0 & 40 & 265 & 1.00 & 1.00 \\
\hline$\leq 2$ & 13 & 124 & $0.69(0.36-1.34)$ & $0.83(0.34-1.73)$ \\
\hline$\geq 3$ & 18 & 83 & $1.44(0.78-2.64)$ & $1.14(0.56-2.34)$ \\
\hline \multicolumn{5}{|l|}{ History of abortion } \\
\hline Yes & 12 & 34 & $2.62(1.28-5.34)$ & $3.45(1.54-7.74)$ ** \\
\hline No & 59 & 438 & 1.00 & 1.00 \\
\hline \multicolumn{5}{|l|}{ Place of delivery } \\
\hline Home & 13 & 196 & $0.32(0.17-0.59)$ & $0.35(0.18-0.68) * *$ \\
\hline Health facilities & 58 & 276 & 1.00 & 1.00 \\
\hline \multicolumn{5}{|l|}{ Mode of delivery } \\
\hline Spontaneous & 57 & 432 & 1.00 & 1.00 \\
\hline Others & 14 & 40 & $2.65(1.36-5.17)$ & $1.71(0.79-3.74)$ \\
\hline \multicolumn{5}{|l|}{ PNC utilization } \\
\hline Yes & 61 & 355 & $2.01(0.99-4.05)$ & $1.46(0.56-3.28)$ \\
\hline No & 10 & 117 & 1.00 & 1.00 \\
\hline \multicolumn{5}{|l|}{ Knowledge of mothers } \\
\hline Good & 47 & 215 & $2.34(1.38-3.95)$ & $1.79(1.10-3.18) *$ \\
\hline Poor & 24 & 257 & 1.00 & 1.00 \\
\hline \multicolumn{5}{|l|}{ Attitude of mothers } \\
\hline Favorable & 54 & 203 & $4.21(2.37-7.48)$ & $3.38(1.83-6.24) * *$ \\
\hline Unfavorable & 17 & 269 & 1.00 & 1.00 \\
\hline \multicolumn{5}{|l|}{ Exposure to media } \\
\hline Yes & 26 & 132 & $1.48(0.88-2.51)$ & $1.06(0.51-1.80)$ \\
\hline No & 45 & 340 & 1.00 & 1.00 \\
\hline
\end{tabular}

** Significantly associated at P-value $<0.01$

* Significantly associated at P-value $<0.05$

\section{Discussion}

Vaccines are the most effective preventive, public health successful means of controlling and eradicating serious diseases (4). Vaccines are more effective when given with recommended time interval; since early 
vaccination can result in failure to generate a protective antibody against the diseases and delayed vaccination leads to more exposure to the diseases $(8,9)$.

The overall childhood vaccination timeliness among children aged 12 to 23 months in Jabitehnan district were $13.1 \%$ (95\% Cl: 10.1-15.8). This implies that the remaining $76.9 \%$ children were susceptible for vaccine preventable diseases; since failure to be vaccinated on time would increase the susceptibility period of children through limiting the herd immunity (27). This finding was higher than the studies done in Menz Lalo district, northeast Ethiopia, $6.2 \%$ (22) and Kenya 6.1\% (20). However, the finding was lower than the results in Pakistan 20.8\% (29), Israel 22\% (30), Ghana 50.5\% (31) and Senegal 72.3\%(32). The possible explanation for this discrepancy might be due to differences in study participant characteristics, study period, design and health service accessibility.

Moreover, only $42.2 \%$ (95\% Cl: $38.1-46.8)$ children were received BCG vaccines timely. It is higher than the findings in Ethiopia (23.9\%) (23) and Bangladesh (24\%) (33). On the contrary, this finding was lower than compared to studies conducted outside in Pakistan (89.3\%) (29), Ghana (88.9\%) (31), and Senegal (88.25\%) (32). Similarly, $42.9 \%$ (95\% Cl: $38.9-47.0)$ children were vaccinated measles vaccine in the recommended time interval.

The finding was higher than the findings in Menz Lalo district, northeast Ethiopia (26.4\%)(22), and Kenya $(28.2 \% 0(20)$. However, this finding was lower than as the studies conducted in Bangladesh (64\%)(33), Ghana (50.5\%) (31) and Senegal (72.3\%) (32). The possible explanation for this discrepancy might be due to the differences in the study participants, access to vaccines, and variations in the recommendation of wastage rate to open BCG and Measles vaccines across countries (22). Besides, this study also revealed that $16.4 \%$ (95\% Cl: $13.3-19.8)$ of children were received measles vaccine earlier than the recommended time interval. The finding was higher than a study conducted in Bangladesh (12\%) (33) and lower than a study done in Pakistan (90\%) (29). The possible justification might be health workers' appointment before nine months of age to prevent open dose vial wastage, mothers/caregivers being too busy on appointment day and forgotten vaccination appointment (22).

The vaccination timeliness was lower in the higher dose of the antigen for those vaccines which had subsequent doses such as penta1-3, OPV 1-3, PCV1-3 and Rota1-2. This might be due to increased maternal/caregiver's workload with other domestic activities while the child gets older and they may not remember vaccination appointments of a child and fear of side effects $(22,34)$.

This study also revealed that 5.5 and $2.6 \%$ of children were received the vaccine earlier than the recommended time interval for Penta 1 and penta3 vaccines, respectively. This finding was in line with a study done in Menz Lalo district, northeast Ethiopia 6.8 and $5.2 \%$ of children were vaccinated Penta 1 and penta3 vaccines, respectively (22). On the other hand, 30 and $53 \%(95 \% \mathrm{Cl}: 49.3-57.6)$ of children aged 12 to 23 months were vaccinated later than the acceptable time interval for Penta1 and penta3 vaccines, correspondingly. This finding was lower than the findings in Menz Lalo district, northeast Ethiopia, 54.1 and $64.5 \%$ of children were received Penta 1 and penta 3 vaccines later than the recommended time interval, respectively (22). However, this finding was higher than the studies done in Pakistan, 19.1 and 
$43.4 \%$ of children received Penta 1 and penta3 vaccines later than the acceptable time interval, correspondingly (29). This discrepancy might be due to mothers/caregivers of children might not remember the appointment date, work load by domestic activities or fear of side effects like fever, pain and swelling.

Rural children were negatively influenced the receiving of vaccines in the recommended age than urban children. This finding was in line with the findings in Ethiopia (23). The possible justification might be due to urban resident mothers might have better information and recognize the importance of vaccination (37).

Children whose mothers gave birth at home were less likely to receive the recommended childhood vaccines in the acceptable time. This finding was supported by the studies conducted in Kenya (11), Ethiopia (23), Pakistan(29), Malawi (38) and Uganda (39). The possible justification might be mothers who gave birth at health facilities might have more opportunity to be informed about child healthcare, including childhood vaccination (29).

Children who were born from educated mothers were more likely to receive their vaccines timely than noneducated mothers. This finding was consistent with the findings of the study done in Bangladesh(33). The possible justification might be educated mothers might have better knowledge about vaccinepreventable diseases and recognize the importance of vaccination.

Children of mothers/caregivers having history of abortion before the birth of the index child were more likely to receive the recommended childhood vaccines with acceptable time interval. This might be due to mothers/caregivers with history of abortion give more emphasis for the health of their child, fear the morbidity of child and understood the burden of childhood infection more than their counterparts.

Children of mothers/caregivers with good knowledge about childhood vaccination were more likely to be vaccinated with the recommended time interval. This is in line with the findings of a study done at Menz Lalo district, northeast Ethiopia (22). This can be suggested mothers/care givers having a better understanding of childhood vaccination schedule, vaccine preventable diseases and reasons for vaccination may bring their child to vaccination site more likely at recommended time. Children of mothers/caregivers with favorable attitude towards childhood vaccination were more likely to be vaccinated timely. This is in line with study conducted in Machakel district, east Gojjam zone (40). This can be suggested mothers/caregivers who had unfavorable attitude about childhood vaccination might not take their child to vaccination site by their appointment.

\section{Limitations of the study}

This cross-sectional study has its own limitations. The study participants were selected based on the presence of immunization cards, which might lead to selection bias because infants whose parents did not keep their immunization cards were excluded from the study. This could be over estimate or underestimate the magnitude of vaccination timeliness. 


\section{Conclusion}

The overall childhood vaccination timeliness status was low compared with the current performance of the vaccination coverage in Ethiopia. Children were received the first dose of OPV, Penta, PCV and Rota vaccines within recommended time relatively higher than the other doses/vaccines. Children received measles vaccine earlier and BCG vaccine later than the acceptable time interval. Variables such as residence, maternal level of education, having history of abortion, place of delivery, knowledge about childhood vaccination and attitudes towards childhood vaccination were affecting vaccination timeliness. Therefore, the policy planners and managers should give emphasis and incorporate vaccination timeliness in the childhood vaccination plan, and better to monitor and evaluate as one potential indicator to enhance the immune status of children. Health planners and managers should also improve women's education and awareness to enhance their attitude towards childhood vaccination to maintain and enhance to bring their children to their health facility in the appointment. Furthermore, it is better to promote institutional delivery service utilization to enhance childhood vaccination timeliness.

\section{Abbreviations}

ANC, Antenatal Care; AOR, Adjusted Odds Ratio; BCG, Bacillus Calmette-Guerin; CDC, Communicable Diseases Control; COR, Crude Odds Ratio; EDHS, Ethiopian Demographic Health Survey; EPI, Expanded Program of Immunization; ETB, Ethiopian Birr; FMOH, Federal Ministry of Health; LMICs, Low and Middle Income Countries; OPV, Oral Polio Vaccine; PCV, Pneumococcal Conjugate Vaccine; PNC, Postnatal Care; SPSS, Statistical Packages for Social Sciences; TT, Tetanus Toxoid; WHO, World Health Organization

\section{Declarations}

\section{Ethics approval and consent to participate}

Ethical approval was obtained from the Ethical Committee of Institute of Public Health, College of Medicine and Health Sciences, University of Gondar (Ref. No. IPH/837/2020). Before the actual data collection was started, concerned body in Jabitehnan Health office was communicated through formal letter issued from the University of Gondar. Official permission and supportive letter were obtained from Jabitehnan health office. Data were anonyms and held on a secure password protected system. Confidentiality during all phases of research activities was kept.

\section{Consent for publication}

Not applicable.

\section{Availability of data and materials}

All the data were included in the study, and data will be available upon a responsible request from the corresponding author. 


\section{Competing Interests}

The authors declare that they have no competing interests.

\section{Funding}

The University of Gondar sponsored this study. However, it has no role in the decision to publish, manuscript preparation, and publication.

\section{Authors' Contributions}

BE designed the study, developed data collection tools, performed the analysis and interpretation of data, and drafted the paper. AD and LY participated in the development of the study proposal, analysis and interpretation, revised drafts of the paper, revised the manuscript. All authors read, revised, and approved the final manuscript.

\section{Acknowledgement}

We would like to give thanks for University of Gondar, College of Medicine and Health sciences, Institute of Public Health, Department of Health Systems and Policy for giving the chance. We forward our gratitude the health officials for their cooperation. We would like to thank study participants for their volunteer participation.

\section{References}

1. Organization WH. State of Inequality: Reproductive Maternal Newborn and Child Health: Interactive Visualization of Health Data: World Health Organization; 2015.

2. Keja K, Chan C, Hayden G, Henderson RH. Expanded programme on immunization. World Health Stat Q. 1988;41(2):59-63.

3. WHO. Immunization today and the next decade. 11. 2018;18.

4. Masters NB, Tefera YA, Wagner AL, Boulton ML. Vaccine hesitancy among caregivers and association with childhood vaccination timeliness in Addis Ababa, Ethiopia. Human vaccines \& immunotherapeutics. 2018;14(10):2340-7.

5. Masters NB, Wagner AL, Boulton MLJHv, immunotherapeutics. Vaccination timeliness and delay in low-and middle-income countries: a systematic review of the literature, 2007-2017. 2019:1-16.

6. World, health, organization(WHO). Recommendations for routine immunization-summary tables. 2019.

7. Hull BP MP. Timeliness of childhood immunisation in Australia. Vaccine 2006;24:4403-8.

8. Akmatov MK, Mikolajczyk RT. Timeliness of childhood vaccinations in 31 low and middle-income countries. Journal of epidemiology and community health. 2012;66(7):e14. 
9. Heininger $U$, Zuberbühler MJEjop. Immunization rates and timely administration in pre-school and school-aged children. 2006;165(2):124-9.

10. Scott $S$ ea. Coverage and timing of children's vaccination: an evaluation of the expanded programme on immunisation in the Gambia. PLoS One. 2014;9(9):e107280.

11. Masters NB, Wagner AL, Carlson BF, Boulton MLJV. Vaccination timeliness and co-administration among Kenyan children. 2018;36(11):1353-60.

12. WHO. United Nations Children's Fund, World Bank: State of the world's vaccines and immunization. 2009.

13. WHO. vaccine coverage 2018.

14. Wahl B, O'Brien KL, Greenbaum A, Majumder A, Liu L, Chu Y, et al. Burden of Streptococcus pneumoniae and Haemophilus influenzae type $b$ disease in children in the era of conjugate vaccines: global, regional, and national estimates for 2000-15. 2018;6(7):e744-e57.

15. Arevshatian L, Clements C, Lwanga S, Misore A, Ndumbe P, Seward J, et al. An evaluation of infant immunization in Africa: is a transformation in progress? Bulletin of the World Health Organization. 2014;85(6):449-57.

16. Machingaidze S, Wiysonge CS, Hussey GDJPm. Strengthening the expanded programme on immunization in Africa: looking beyond 2015. 2013;10(3):e1001405.

17. FMOH. mortality and morbidity of children in ethiopia 2018.

18. WHO. Ethiopia Disease Outbreaks: Chikungunya, Cholera, Measles, Poliovirus. 2019.

19. CDC. General Best Practice Guidelines for Immunization: Timing and Spacing of Immunobiologics.:11-40.

20. Adetifa IM, Karia B, Mutuku A, Bwanaali T, Makumi A, Wafula J, et al. Coverage and timeliness of vaccination and the validity of routine estimates: Insights from a vaccine registry in Kenya. 2018;36(52):7965-74.

21. Nakatudde I, Rujumba J, Namiiro F, Sam A, Mugalu J, Musoke PJPo. Vaccination timeliness and associated factors among preterm infants at a tertiary hospital in Uganda. 2019;14(9).

22. Marefiaw TA, Yenesew MA, Mihirete KMJPo. Age-appropriate vaccination coverage and its associated factors for pentavalent 1-3 and measles vaccine doses, in northeast Ethiopia: A community-based cross-sectional study. 2019;14(8).

23. Boulton ML, Carlson BF, Wagner AL, Porth JM, Gebremeskel B, Abeje Y. Vaccination timeliness among newborns and infants in Ethiopia. PLoS One. 2019;14(2):e0212408.

24. Central, Statistics, Agency, (CSA). National Population Statistics. Federal Democratic Republic of Ethiopia, Central Statistical Authority, Addis Ababa. 2007.

25. Klett-Tammen CJ, Krause G, Seefeld L, Ott JJ. Determinants of tetanus, pneumococcal and influenza vaccination in the elderly: a representative cross-sectional study on knowledge, attitude and practice (KAP). BMC Public Health. 2016;16:121. 
26. Picchio CA, Carrasco MG, Sague-Vilavella M, Rius C. Knowledge, attitudes and beliefs about vaccination in primary healthcare workers involved in the administration of systematic childhood vaccines, Barcelona, 2016/17. Euro surveillance : bulletin Europeen sur les maladies transmissibles $=$ European communicable disease bulletin. 2019;24(6).

27. Hu Y, Chen Y, Guo J, Tang X, Shen L. Completeness and timeliness of vaccination and determinants for low and late uptake among young children in eastern China. Human vaccines \& immunotherapeutics. 2014;10(5):1408-15.

28. $\mathrm{FMOH}$. Ethiopian national expanded program on immunization comprehensive multi-year plan 2016-2020,. 2016-2020.

29. Noh J-W, Kim Y-m, Akram N, Yoo KB, Cheon J, Lee LJ, et al. Determinants of timeliness in early childhood vaccination among mothers with vaccination cards in Sindh province, Pakistan: a secondary analysis of cross-sectional survey data. 2019;9(9):e028922.

30. Stein-Zamir C, Israeli A. Timeliness and completeness of routine childhood vaccinations in young children residing in a district with recurrent vaccine-preventable disease outbreaks, Jerusalem, Israel. Eurosurveillance. 2019;24(6).

31. Laryea DO, Abbeyquaye Parbie E, Frimpong E. Timeliness of childhood vaccine uptake among children attending a tertiary health service facility-based immunisation clinic in Ghana. BMC Public Health. 2014;14:90.

32. Mbengue MAS, Mboup A, Ly ID, Faye A, Camara FBN, Thiam M, et al. Vaccination coverage and immunization timeliness among children aged 12-23 months in Senegal: a Kaplan-Meier and Cox regression analysis approach. The Pan African medical journal. 2017;27(Suppl 3):8.

33. Sheikh N, Sultana M, Ali N, Akram R, Mahumud R, Asaduzzaman M, et al. Coverage, Timelines, and Determinants of Incomplete Immunization in Bangladesh. Tropical medicine and infectious disease. 2018;3(3):72.

34. Tefera YA, Wagner AL, Mekonen EB, Carlson BF, Boulton ML. Predictors and barriers to full vaccination among children in Ethiopia. Vaccines. 2018;6(2):22.

35. Awofeso N, Rammohan A, lqbal KJBPH. Age-appropriate vaccination against measles and DPT-3 in India-closing the gaps. 2013;13(1):358.

36. Bailly AC, Gras P, Lienhardt JF, Requillart JC, Vie-le-Sage F, Martinot A, et al. Timeliness of vaccination in infants followed by primary-care pediatricians in France. Human vaccines \& immunotherapeutics. 2018;14(4):1018-23.

37. Debie A, Lakew AM. Factors associated with the access and continuum of vaccination services among children aged 12-23 months in the emerging regions of Ethiopia: evidence from the 2016 Ethiopian demography and health survey. Italian Journal of Pediatrics. 2020;46(1):1-11.

38. Mvula H, Heinsbroek E, Chihana M, Crampin AC, Kabuluzi S, Chirwa G, et al. Predictors of Uptake and Timeliness of Newly Introduced Pneumococcal and Rotavirus Vaccines, and of Measles Vaccine in Rural Malawi: A Population Cohort Study. PLoS One. 2016;11(5):e0154997. 
39. Babirye JN, Engebretsen IM, Makumbi F, Fadnes LT, Wamani H, Tylleskar T, et al. Timeliness of childhood vaccinations in Kampala Uganda: a community-based cross-sectional study. 2012;7(4):e35432.

40. Yenit MK, Gelaw YA, Shiferaw AM. Mothers' health service utilization and attitude were the main predictors of incomplete childhood vaccination in east-central Ethiopia: a case-control study. Archives of Public Health. 2018;76(1):14. 